Received: 8 August 2017

Accepted: 27 November 2017

Published online: 07 December 2017

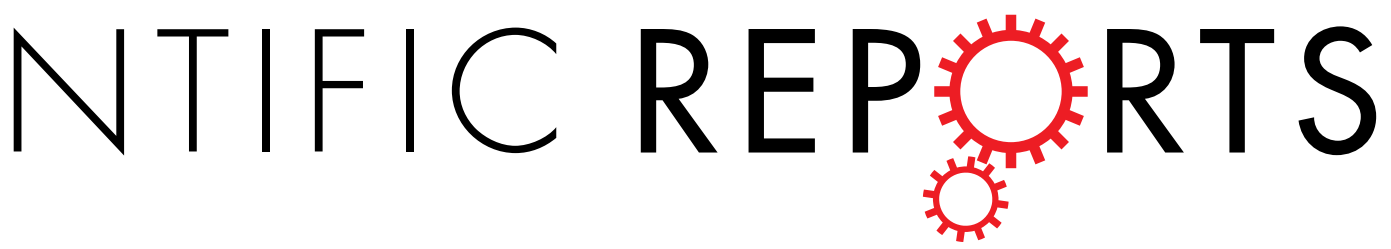

\title{
Integrated DNA methylome and transcriptome analysis reveals the ethylene-induced flowering pathway genes in pineapple
}

Jiabin Wang ${ }^{1,2,3}$, Zhiying $\mathrm{Li}^{1,2,3}$, Ming Lei ${ }^{1,2,3}$, Yunliu Fu ${ }^{1,2,3}$, Jiaju Zhao ${ }^{1,2,3}$, Mengfei Ao ${ }^{1,2,3}$ \& $\operatorname{LiX} \mathbf{u}^{1,2,3}$

Ethylene has long been used to promote flowering in pineapple production. Ethylene-induced flowering is dose dependent, with a critical threshold level of ethylene response factors needed to trigger flowering. The mechanism of ethylene-induced flowering is still unclear. Here, we integrated isoform sequencing (iso-seq), Illumina short-reads sequencing and whole-genome bisulfite sequencing (WGBS) to explore the early changes of transcriptomic and DNA methylation in pineapple following high-concentration ethylene (HE) and low-concentration ethylene (LE) treatment. Iso-seq produced 122,338 transcripts, including 26,893 alternative splicing isoforms, 8,090 novel transcripts and 12,536 candidate long non-coding RNAs. The WGBS results suggested a decrease in CG methylation and increase in $\mathrm{CHH}$ methylation following $\mathrm{HE}$ treatment. The $\mathrm{LE}$ and $\mathrm{HE}$ treatments induced drastic changes in transcriptome and DNA methylome, with LE inducing the initial response to flower induction and $\mathrm{HE}$ inducing the subsequent response. The dose-dependent induction of FLOWERING LOCUST-like genes (FTLs) may have contributed to dose-dependent flowering induction in pineapple by ethylene. Alterations in DNA methylation, IncRNAs and multiple genes may be involved in the regulation of $F T L s$. Our data provided a landscape of the transcriptome and DNA methylome and revealed a candidate network that regulates flowering time in pineapple, which may promote further studies.

Pineapple (Ananas comosus L.) is an economically significant tropical fruit of the bromeliads. Natural flowering in pineapple is commonly induced by short-day photoperiods, cool night temperatures and dry weather, and other stresses, such as root damage, can also trigger flowering ${ }^{1}$. Ethylene-induced flowering is widely used to synchronize pineapple flowering, facilitating year-round production.

The transition from vegetative growth to flowering is a crucial developmental change for flowering plants. This transition occurs in response to various environmental and endogenous cues. In Arabidopsis, flowering time is controlled by age-, autonomous-, sugar budget-, gibberellin-, ambient temperature-, photoperiod- and vernalization-dependent pathways ${ }^{2-4}$. Floral integrator genes such as $F T, S O C 1$, and $A G L 24$, converge various cues and activate the floral identity genes (e.g., $L F Y, A P 1, S E P 3$ and $F U L$ ), which confer the transition to the floral meristem ${ }^{5}$.

Ethylene is a plant hormone that regulates plant growth and development, as well as responses to biotic and abiotic stresses ${ }^{6,7}$. The role of ethylene in the flowering transition is unclear, as ethylene can both promote and delay flowering in both Arabidopsis and rice under different conditions ${ }^{8-11}$. In pineapple, flowering is triggered by a burst of ethylene production in response to various cues, in which the AcACS2 plays an important role ${ }^{12}$. Four ethylene receptors genes (AcERS1b, AcERS1b, AcETR $2 a$, and AcETR2b) have been cloned, and expression analysis showed that $A c E R S 1 b, A c E T R 2 a$, and $A c E T R 2 b$ play key roles in pineapple flowering ${ }^{13}$. Ectopic overexpression of AcPISTILLATA (PI) and AcFT induces early flowering in Arabidopsis; however, PISTILLATA and FT expression

${ }^{1}$ Institute of Tropical Crop Genetic Resources, Chinese Academy of Tropical Agricultural Sciences, Danzhou, 571737, Hainan, China. ${ }^{2}$ Ministry of Agriculture Key Laboratory of Crop Gene Resources and Germplasm Enhancement in Southern China, Danzhou, 571737, Hainan, China. ${ }^{3}$ Hainan Province Key Laboratory of Tropical Crops Germplasm Resources Genetic Improvement and Innovation, Danzhou, 571737, Hainan, China. Jiabin Wang and Zhiying Li contributed equally to this work. Correspondence and requests for materials should be addressed to L.X. (email: xllizhiying@vip.163.com) 

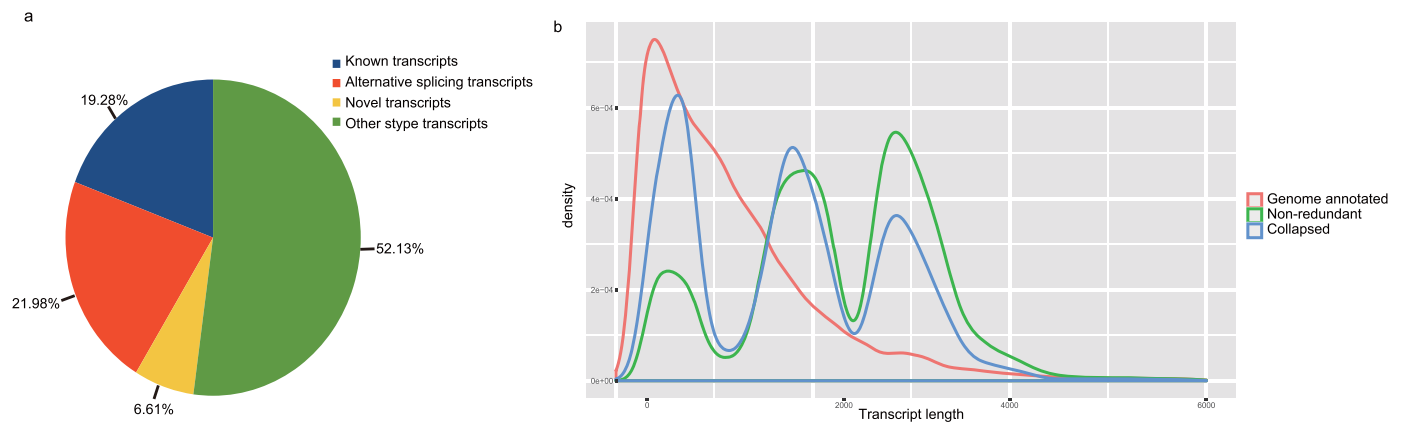

Figure 1. Summary of high-quality transcripts obtained using iso-seq. (a) Distribution of high-quality transcripts. (b) Length distribution of annotated pineapple genes and high-quality transcripts. Gene models: Pineapple genome annotated gene; Non-redundant transcripts: high-quality transcripts perfectly mapped to the genome; un-collapsed transcripts: transcripts unmapped or insufficiently mapped to the genome.

peaked on day 40 after ethylene treatment when the fruit and floral organs were forming, indicating an important role in floral organ and fruit development ${ }^{14,15}$. Liu et al. (2016) utilized Illumina sequencing to integrate transcriptomic changes shoot apical meristems of floral buds in response to ethylene ${ }^{16}$, indicating that LTI, FT, and VRN1 involved in the process of floral development. The ethylene level of plants were increased and the $\mathrm{GA}_{3}$ level were decreased during first 24 hours after flowering forcing using ethephon, coupling with the upregulation of GA2ox1 and $P I^{17}$. And 48 hours after treatment, the shoot apex showed differentiation signs and plants can been seen "open heart" 2 weeks after treatment ${ }^{1,17}$. However, further research is needed to understand the molecular mechanism regulating the transition from vegetative to reproductive growth of pineapple during forcing flowering.

DNA methylation is a widely studied epigenetic modification that occurs at $\mathrm{CG}, \mathrm{CHG}(\mathrm{H}=\mathrm{A}, \mathrm{T}$ or $\mathrm{C})$ and $\mathrm{CHH}$ sites through the covalent addition of a methyl group to cytosine in plants. DNA methylation plays a key role in plant development and stress responses ${ }^{18-22}$. Moreover, DNA methylation is involved in controlling flowering time. Silencing of the FWA gene by DNA methylation controls flowering time in Arabidopsis ${ }^{22}$. Treatment with 5-azaC, a cytosine methyltransferase inhibitor, induces flowering in Arabidopsis, wheat and Pharbitis nil ${ }^{23-25}$.

With the publication of the genomes of the pineapple varieties $\mathrm{F} 135$ and $\mathrm{MD}-2$ genome, mapping genomic DNA methylation and transcriptomic changes in inducing flowering is now possible ${ }^{26,27}$. After treating plants with water, a low concentration of ethephon $(600 \mathrm{mg} / \mathrm{L})$ and high concentration of ethephon $(1200 \mathrm{mg} / \mathrm{L})$ 24 hours, we utilized PacBio RS II and Sequel iso-seq, Illumina short-reads sequencing and whole-genome bisulfite sequencing (WGBS) to track changes in the transcriptome and DNA methylome, which triggering the floral transition. The results implied that the DNA methylation level, alternative splicing (AS), alternative polyadenylation (APA) and the expression levels of multiple genes were changed in response to ethylene, which may contribute to ethylene-induced flowering.

\section{Results}

Transcriptome sequencing using iso-seq and isoform detection. To detect transcriptomic changes during flowering induction, four libraries with $<1-, 1-2,2-3$, and $>3 \mathrm{k}$ fragment sizes were constructed and sequenced using PacBio RS II and Sequel, yielding 1,068,545 ROI reads in total (Supplementary Table S1). Of the ROI reads, 774,999 were $5^{\prime}$ primer observed reads, $1,081,410$ were $3^{\prime}$ primer observed reads, 856,009 were poly A tail reads and 725,933 were full-length non-chimeric reads. Using Quiver, the full-length reads were polished and classified as 122,338 high-quality and 234,917 low-quality reads (Supplementary Table S2).

After polishing, 122,338 high-quality reads were mapped to the genome using GMAP, with $99.16 \%$ of reads mapped. Then, the reads mapped to the genome with high quality were collapsed into non-redundant transcripts, which resulted in 45,876 transcripts. Figure $1 \mathrm{~b}$ shows the length distribution of annotated genes in the pineapple genome, perfectly mapped transcripts, and insufficiently mapped and unmapped transcripts, with mean lengths of $1,171,1,857$ and 1,695 bp, respectively. Using Cuffcompare, the mapped reads were compared to the annotated gene model. The reads mapped to 22,676 loci, including 7,673 (33.90\%) novel loci. Through AS analysis, we identified 29,801 isoforms from 13,264 loci, of which 9,371 isoforms matched the intron chain of genome annotation genes and 20,140 alternative splicing isoforms (Fig. 1a). A total of 71 transcripts mapped to 34 miRNA genes, and 174 transcripts mapped to 39 phasiRNA genes. An additional 352 transcripts mapped to 140 transposable elements (TEs), and 726 reads mapped to rRNA genes.

Transcriptome sequencing using Illumina short reads. Sequencing identified 26,870,796, 29,999,998, $27,224,962,29,526,890,29,688,904,26,878,05230,487,590,30,085,504$, and 29,442,846 bp for the 9 samples of the CK, LE and HE groups, with three biological replicates each (Supplementary Table S3). The clean reads had a $59.05 \%$ genome-map rate and a $47.29 \%$ gene-map rate. Using the short reads, we detected the expression of $23,030(89.36 \%)$ annotated genes.

Alternative splicing and alternative polyadenylation. Of the 28,901 isoforms, AS events were divided into five categories: 1) 827 transcripts with a $3^{\prime}$ AS site; 2) 780 transcripts with a $5^{\prime}$ AS site; 3) 6,746 transcripts with exon skipping; 4) 16,917 transcripts with intron retention; and 5) 8,408 transcripts with sizes of exon disagreement (Fig. 2a). A total of 7,767 genes had more than one AS isoform (Fig. 2b). Given the APA of mRNA, 
a
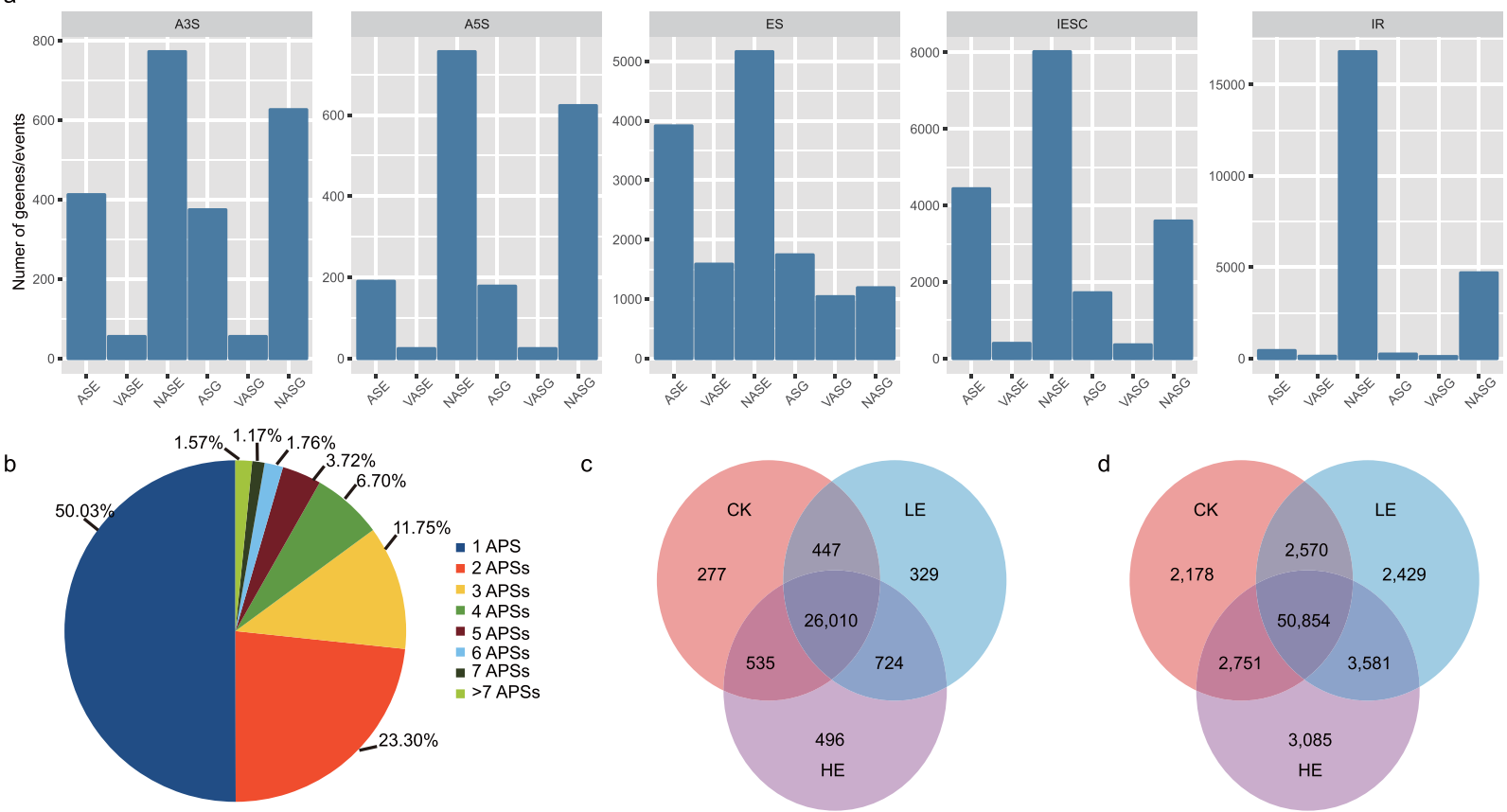

Figure 2. Summary of alternative splicing isoforms and alternative adenylation isoforms. (a) Statistics of the numbers of alternative splicing isoforms of genes. A3S: alternative $3^{\prime}$ splice site; A5S: alternative $5^{\prime}$ splice site; ES: exon skipping; IESC: internal exon size change; IR: intron retention. ASE: alternative splicing events annotated in pineapple genome; VASE: the iso-seq reads validated alternative splicing events annotated in pineapple genome; NASE: the new alternative splicing events arose; ASG: ASE related genes; VASG: VASE related genes; NASG related genes. (b) Statistics of the number of alternative adenylation isoforms of genes. (c) The distribution of alternative splicing events. (d) The distribution of alternative splicing isoforms in CK, LE and HE. (e) The distribution of alternative adenylation isoforms in CK, LE and HE.

6,604 of 13,216 loci had more than one alternative polyadenylation site (APS). Using short-read quantification, we identified 277 CK-specific, 329 LE-specific and 496 HE-specific AS isoforms, with 2,178 CK-specific, 2,429 LE-specific and 3,085 HE-specific isoforms (Fig. 2c and d).

The pineapple genome contains 1,601 annotated transcription factor (TF) loci ${ }^{28}$. The iso-seq reads mapped to 780 loci with 1,629 isoforms. Of the TF loci, 352 loci have multiple isoforms. A total of 12 CK-specific, 21 HE-specific and 22 LE-specific isoforms, as well as 96 ethylene-induced isoforms, were detected. A total of 2,568 APS of 619 loci were identified, with $74 \mathrm{CK}$-specific, $103 \mathrm{LE}$-specific and $125 \mathrm{HE}$-specific isoforms, as well as 329 ethylene-induced APS isoforms.

Novel transcripts and IncRNAs. By predicting the coding potential of novel transcripts, we obtained 12,536 candidate lncRNAs and 8,090 candidate novel coding transcripts. The novel coding transcripts were aligned to the NR database, with 846 transcripts hitting known genes (Supplementary Dataset S1). According their position relative to the gene annotations, the lncRNAs were classified into five groups: 1,182 (76.00\%) in the sense strand, 2,382 (6.28\%) in the antisense strand, 365 (2.70\%) in intronic regions, 2,736 (7.25\%) in intergenic regions and 5,871 (7.76\%) unmapped or insufficiently mapped to the genome (Fig. 3b). The lncRNAs ranged in length from 280 to 6,025, with a mean length of 1,860. Of the lncRNAs mapped pineapple genome, 5,517 lncRNAs had a single exon, 745 lncRNAs had two exons and $405 \operatorname{lncRNAs}$ had more than two exons (Fig. 3a). LncRNAs were expressed in a lower level with median 0.69 and mean 21.74 FPKM value (Fig. 3c). And 3,700 lncRNAs were with 0 FPKM values, which were not detected by short reads. Using the quantification of short reads, we identified 293, 304, and 504 CK-, LE- and HE-specific lncRNAs, respectively (Fig. 3d). To determine the function of lncRNAs involved in flowering induction, we predicted the candidate targets of 298 lncRNAs in cis. The flowering related genes FTL1, BHLH93, GA2OX8, MYB30 and EIL1 were targets of lncRNAs.

Differential expression analysis. After quantification of short reads using RSEM, we identified differentially expressed genes (DEGs) and transcripts using NOISeq. We identified 935, 833 and 799 upregulated and 674, 710 and 846 downregulated genes in CK_vs_LE, CK_vs_HE and LE_vs_HE, totaling 2,978 genes. We also performed differential expression analysis on protein-coding mRNA isoforms and lncRNAs. In total, 4,647 isoforms from 2,655 loci were differentially expressed in response to ethylene, with 993, 1,430, 1166 upregulated and 915, 1490, 1199 downregulated isoforms in CK_vs_LE, CK_vs_HE and LE_vs_HE, respectively. Among lncRNAs, 61, 87 and 68 were upregulated, and 56, 91 and 72 were downregulated, totaling 294 lncRNAs. From the DEGs, we selected 18 protein-coding mRNAs and 14 lncRNAs for qRT-PCR validation. The expression patterns of most genes were similar to the expression patterns quantified using short reads (Supplementary Figs S1 and 2). 
a

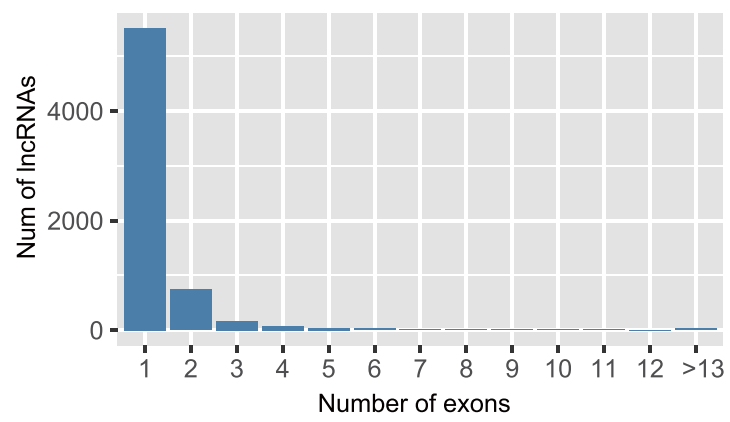

C

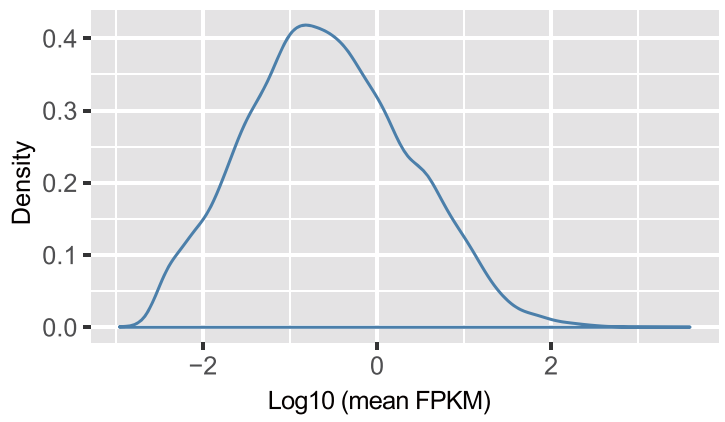

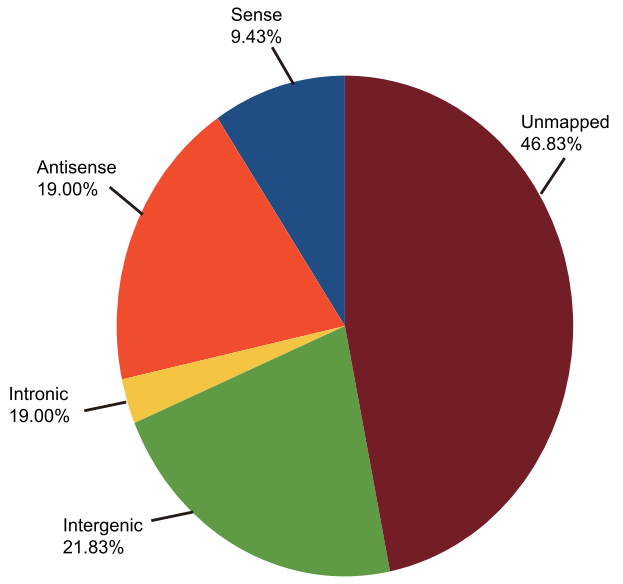

d

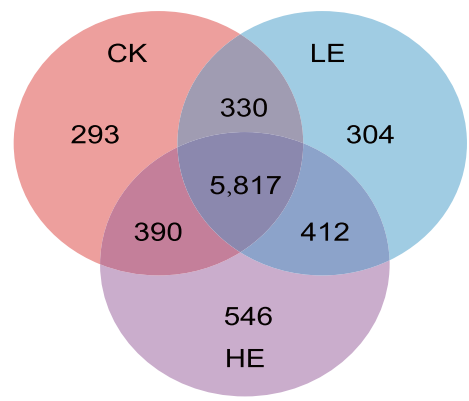

Figure 3. Summary of lncRNAs. (a) The distribution of the number of exons in lncRNAs. (b) The distribution of five types of lncRNAs. (c) The mean expression level lncRNAs. The expression level were normalized use $\log 10$ (mean FPKM). (d) The distribution of lncRNAs in CK, LE and HE.

Co-expression network analysis. Using WGCNA, we predicted the co-expression network of the annotated pineapple genes, and the genes were classified into 96 clusters. The interactions of each cluster were predicted using SPINE (Supplementary Fig. S3). LE and HE separately interacted with 11 clusters each. Six clusters ('darkolivergreen4', 'darkturquoise', 'navajowhite1', 'paleturquoise,' 'plum2' and 'saddlebrown'), containing 677 genes, negatively interacted with HE, and 5 clusters ('pink, 'skyblue', 'skyblue3', 'turquoise', and 'yellow4'), containing 5852 genes, positively interacted with HE, representing the genes responding HE. Seven clusters ('blue', 'brown', 'darkturquoise', 'darkmagenta,' 'firebrick4', 'green' and 'skyblue'), containing 5716 genes, positively interacted with LE, and four clusters ('corall', 'purple', 'steelblue' and 'violet'), containing 802 genes, negatively interacted with LE, representing the genes responding to LE. The 'darkturquoise' cluster interacted positively with LE but negatively with HE. And the clusters 'blue', 'pink', 'grey60', 'magenta' interacted negatively with CK, representing the genes whose expression changed under LE or HE treatment. A subnetwork of candidate flowering genes comprising 87 nodes and 728 edges was extracted (Fig. 4 and Supplementary Dataset S2). Genes in the 'brown' and 'blue' nodes positively interacted with LE, genes in the 'turquoise, 'pink', 'yellow4' and 'skyblue3' nodes positively interacted with 'HE', and genes in the 'paleturquoise' and 'saddlebrown' nodes negatively interact with 'HE', while the else genes negatively interact CK. The networks included 5 FTLs, which may be regulated by the other genes in the network. FTL4 positively interacted with LE, while FTL1, FTL2 and FTL9 positively interacted with HE. Only FTL1 was expressed in CK, while FTL1, FTL2 and FTL9 were induced by HE. Homeostasis among these factors represses and promotes flowering, which ensures the repression of FTL1, FTL2 and FTL9 and the induction of FTL4 in LE and CK and the induction of FTL1, FTL2 and FTL9 and the repression of FTL4 in HE. The candidate genes involved in the regulation of TFLs may relate to the circadian rhythm and stress responses. The genes in the subnetwork can be divided into three categories: genes whose expression was more significantly altered with LE than with HE; genes whose expression was more significantly altered with HE than with LE; genes whose expression was altered by HE and LE but did not differ significantly between the two. In other words, changes in the expression of genes in the three categories in LE and HE may be successive processes that ultimately results the induction of FTL4. According to the DISCERN score, the level of topological alternation of gene networks in different conditions, TOE3 (LOC109711971), NAC35 (LOC109713884) and JMJ30 (LOC109725417) were the top-scoring genes in LE_vs_HE that were involved in FTL repression.

Landscape of genomic DNA methylation. Three pineapple samples under CK, HE, LE treatment was subjected to WGBS. The results generated 29,592,403,200, 27,528,550,600, and 22,905,508,800 bp of clean data, covering $85.36 \%, 85.07 \%$, and $85.75 \%$ of the genome for CK, HE, and LE. Furthermore, 84.78\% (83.67\% CG, 


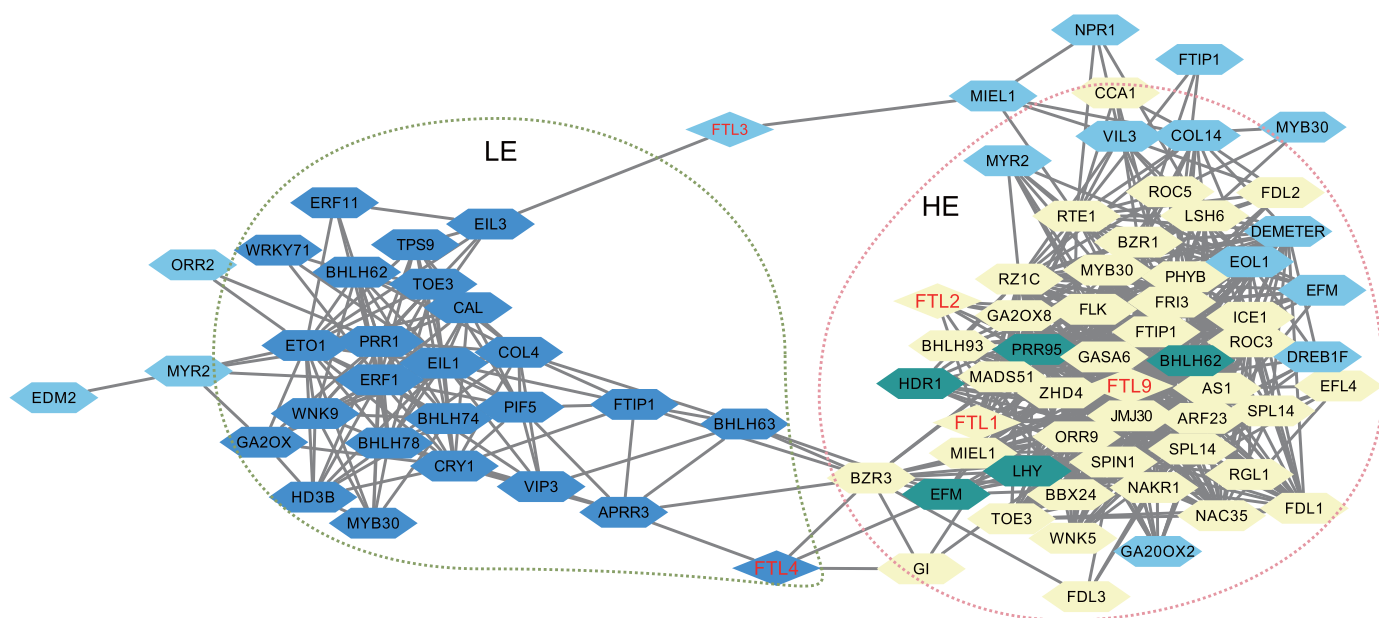

Figure 4. The co-expression network of flowering-related genes. FTLs are shown in red. The different colors of the nodes represent gene clusters: yellow, clusters showing a positive response to HE; green, clusters showing a negative response to HE; steelblue, clusters with a positive response to LE; blue, clusters with no significant response to $\mathrm{HE}$ or LE.

85.21\% CHG, 84.65\% CHH), 83.44\% (82.59\% CG, 84.28\% CHG, 83.48\% CHH), and 85.26\% (84.07\% CG, 85.62\% $\mathrm{CHG}$, and $85.48 \% \mathrm{CHH}$ ) of Cs were mapped with reads in the CK, LE and HE groups.

In total, 14,584,760, 13,721,730 and 14,685,198 mCG; 8,957,987, 8,455,848 and 9,182,888 mCHG; and $11,916,174,13,859,070$ and 17,146,463 $\mathrm{mCHH}$ were detected in the CK, LE and HE groups, respectively (Fig. 5a). These results suggested that LE and HE differentially altered genomic DNA methylation patterns. The numbers of $\mathrm{mCG}$ and $\mathrm{mCHGs}$ decreased following LE treatment but increased following HE treatment. The increase in the number of mCHHs was also much higher in HE than in LE.

The $\mathrm{mCs}$ in each context were further classed as constitutive, LE-specific, HE-specific and varied (Fig. 5b). Constitutive methylated $\mathrm{mC}$ decreased in the order of $\mathrm{mCG}, \mathrm{mCHG}$ and $\mathrm{mCHH}$, indicating that the pathways mediating $\mathrm{mCG}, \mathrm{mCHG}$ and $\mathrm{mCHH}$ methylation responded differentially to $\mathrm{HE}$ and LE.

Furthermore, to detect the effects of DNA methylation changes on genes, the average methylation level of the upstream, gene body and downstream were calculated (Fig. 5e). The DNA methylation level decreased in order of CG, $\mathrm{CHG}$ and $\mathrm{CHH}$. In upstream, the $\mathrm{CHG}$ and $\mathrm{CHH}$ methylation level is lower than in gene downstream and body. Compared with $\mathrm{CK}, \mathrm{mCHG}$ and $\mathrm{mCHH}$ increased in the $\mathrm{HE}$ and LE groups, and mCG increased with $\mathrm{LE}$ and decreased with $\mathrm{HE}$, indicating that ethylene treatment significantly altered the average methylation level (Fig. 5d). In particular, $\mathrm{mC}$ levels in the $5^{\prime}$ and $3^{\prime}$ UTR was more significantly altered than other gene regions.

According the mean methylation level of gene body and upstream, the genes were classed into 4 clusters: Cluster 1 contained genes that had high level CG methylation in upstream; cluster 2 contained genes that had higher CG, CHG and CHH methylation level in gene body than in upstream; cluster 3 contained genes that had lower methylation level in general; cluster 4 contained genes that had high level CG and CHG methylation in both gene body and upstream, but low $\mathrm{CHH}$ methylation in gene body and upstream (Supplementary Fig. S4A-D). The 4 clusters had 7209, 7277, 6878 and 2275 genes, separately, of which the mean expression level of cluster 4 was significantly lower than other clusters (Supplementary Fig. S4E).

DMR identification and stats. Using methylPipe, DMRs between CK and LE (CK_vs_LE), between CK and HE (CK_vs_HE), and between HE and LE (LE_vs_HE) were identified. A total of 4,941, 2,563 and 6047 DMRs were identified for CK_vs_HE, 3,458, 1,764 and 5,462 for CK_vs_LE, and 6,740, 3,769 and 6,714 for LE_ vs_HE in the contexts of CG, CHG and CHH (Fig. 5c). It's thought that DMRs for CK_vs_LE represented the initial alterations in flowering inducing, LE_vs_HE represented for the subsequent alterations and CK_vs_HE represented the ultimate alterations that required for flowering. In total, 4,210, 2,156 and 5,316 DMRs in contexts of CG, CHG and CHH arose in CK_vs_HE but not in CK_vs_LE, and 734, 305 and 1,071 DMRs in contexts of CG, CHG and CHH arose in both CK_vs_HE and CK_vs_LE, which were the flowering inducing related DMRs. About 50\% DMRs in the contexts of CG and CHG are overlapped, indicating the co-regulation of DNA methylation in the contexts of CG and CHG.

Along with DMRs for CG and CHG in CK_vs_HE, the number of hypo DMRs exceeded the number of hyper DMRs, suggesting that ethylene treatment prefers hypo methylation during flowering induction, while the DMRs for $\mathrm{CHH}$ prefers hyper methylation. In addition, approximately $33.31 \%, 3.38 \%$ and $63.30 \%$ of DMRs were distributed in genes, TEs and intergenic regions. In total, the upstream region of 4,336 genes and the coding region of 3,684 genes showed overlapping DMRs in the mCG, mCHG and $\mathrm{mCHH}$ contexts in response to HE and LE.

The regulation of FT-like genes. Nine FT-like genes were annotated in the pineapple genome. Phylogenetic analysis of these FT genes and FT-like genes from other organisms divided the 9 FT-like genes into five groups (Fig. 6a). FTL1, FTL2, FTL8 and FTL9 were grouped with ZmZCN8, which functions as a flowering activator $^{29}$. FTL5 was positioned in the group containing OsRFT1, *AcFT1 and *AcFT2, which also function as 


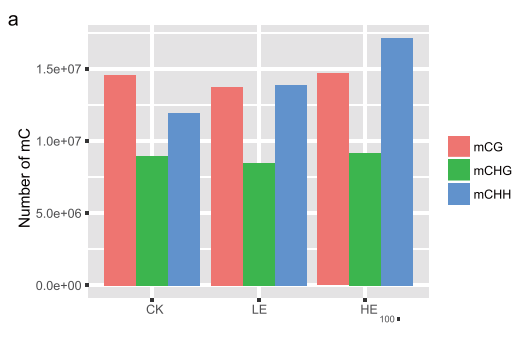

d

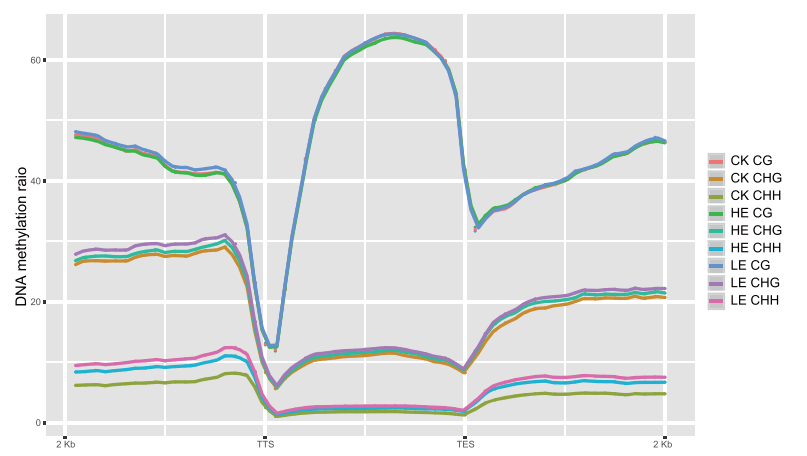

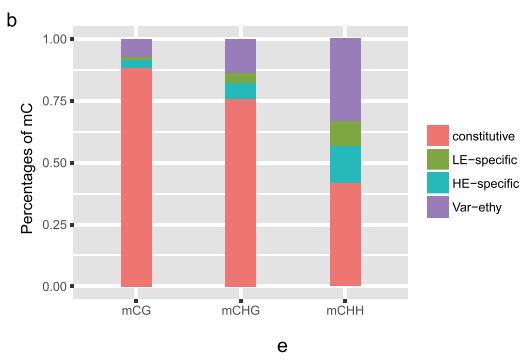
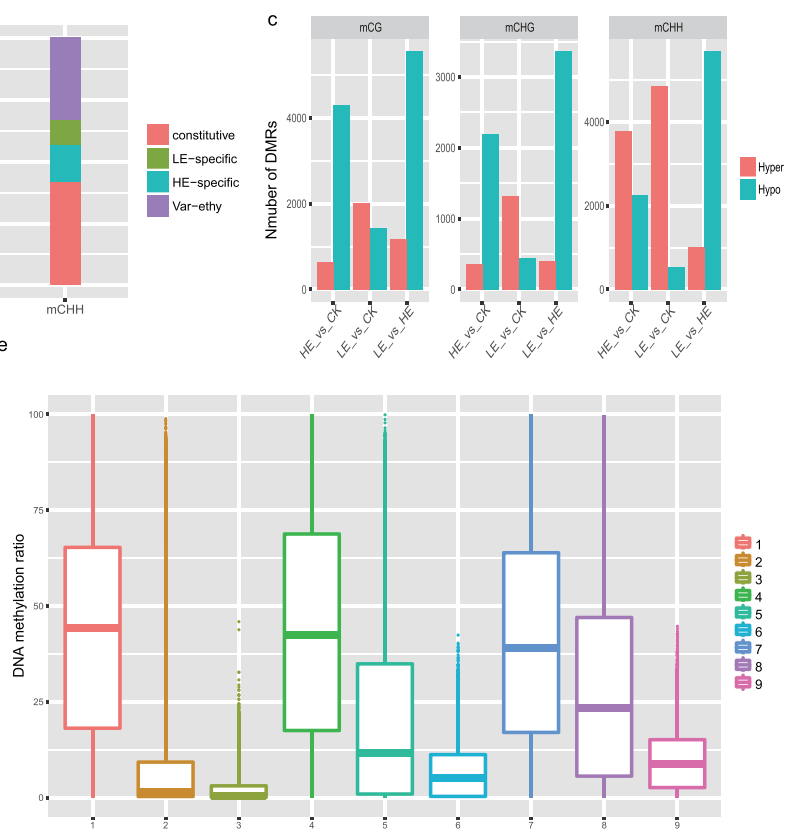

Figure 5. Summary of DNA methylation. (a) Statistics on the numbers of mCs. (b) The distribution of mCs in CK, LE and HE. (c) The statistics of DMRs. (d) The mean DNA methylation level of in the different gene regions. (e) The box-plot of DNA methylation level in the different gene region. 1: CG methylation in gene upstream; 2: CHG methylation in gene upstream; 3: $\mathrm{CHH}$ methylation in gene upstream; 4: CG methylation in gene downstream; 5: CHG methylation in gene downstream; 6: $\mathrm{CHH}$ methylation in gene downstream; 7: CG methylation in gene body; 8: CHG methylation in gene body; 9: $\mathrm{CHH}$ methylation in gene body.

a

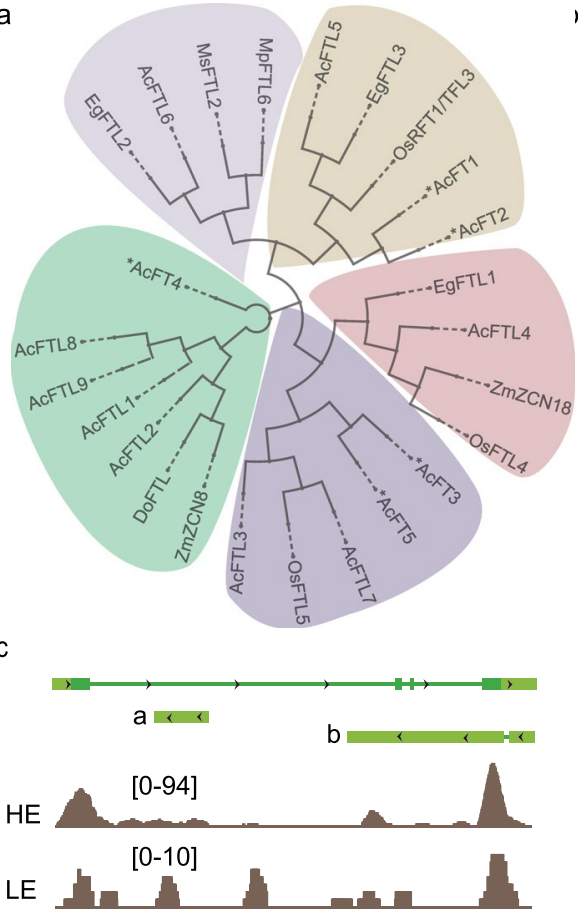

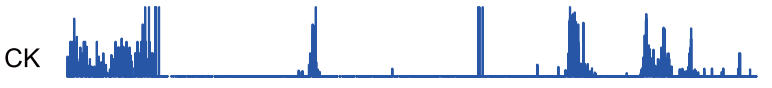
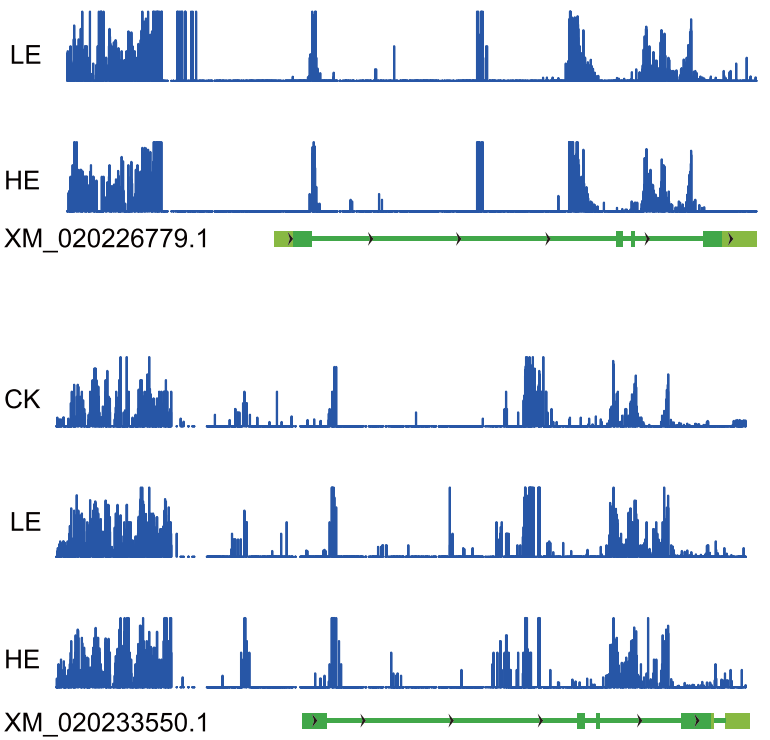

Figure 6. Summary of FTLs. (a) Phylogram of different FLT-like genes from monocots. Ac: Ananas comosus; *Ac: Allium cepa; Os: Oryza sativa Japonica Group; Mp: Musa acuninata AAA Group; Ms: Musa AAB group; Eg: Elaeis guineensis; Zm, Zea mays; Do: Dichanthelium oligosanthes. (b) The CHH methylation type of FTL1 transcripts XM_020233550.1 and FTL9 transcripts XM_020226779.1. (c) The location and expression of two lncRNAs from FTL9. a: the lncRNA c10180/f1p1/463; b: the lncRNA c19722/f1p1/1757. 
flowering activators ${ }^{30,31}$. FTL4 is positioned in the group containing ZmZCN18, which is constitutively expressed in leaves ${ }^{29}$. FTL8, which does not have a complete ORF, is predicted to be a lncRNA ${ }^{32}$.

We observed the CHH methylation level were increased in FTL1 and FTL9 gene upstream and body upon LE treatment (Fig. 6b). But the CHH methylation of FTL9 was decreased upon HE treatment compared to LE treatment. For the last exon region of FTL1, CG methylation level was increased upon HE treatment. The significantly different DNA methylation alteration may contribute to the different expression pattern of FTL1 and FTL9.

lncRNA prediction identified two antisense lncRNAs (c10180/f1p1/463 and c19722/f1p1/1757) from the FTL9 locus (Fig. 6c) and one antisense lncRNA (c494294/f1p1/2025) from the FTL4 locus. The lncRNA c10180/ f1p1/463 was induced by both HE and LE, while the expression of c19722/f1p1/1757 was higher in HE than in LE. The expression of c494294/f1p1/2025 increased in LE and decreased in HE. The three antisense lncRNAs may be involved in regulating FTL9 and FTL4 in cis.

\section{Discussion}

The iso-seq results provided a comprehensive landscape of full-length isoforms. Using the obtained 122,338 HQ reads, we identified 28,901 isoforms of the annotated gene model. Given isoforms with different APS, we identified 6,604 loci with one or more APS. Using short-reads quantification, we identified CK, LE and HE-specific AS and APA isoforms, which implied changes in the complexity of the transcriptome in response to LE and HE treatments. Through differential expression analysis of AS and APA isoforms, we identified some isoforms of genes that had different expression patterns from those of other isoforms, which may have different functions. Importantly, the identified novel 8,090 genes and the isoforms of 7,756 loci had no transcripts that equally matched to the annotated transcripts, which indicated great improvement of the gene model annotations.

lncRNAs are major components of the transcriptome and play a key role in different biological processes. In particular, lncRNAs have also been shown to participate in flowering by mediating the epigenetic silencing of FLC in vernalization ${ }^{33}$. In our study, we predicted 12,536 candidate lncRNAs, which is close to the number of candidate $\operatorname{lncRNAs}$ in maize $(12,226)^{34}$. Of the 2,357 candidate $\ln$ CRNAs annotated in pineapple, $458 \operatorname{lncRNAs}$ we identitied in our studies. For low-expression lncRNAs that were filtered out for differential expression analysis, we identified 294 differentially expressed lncRNAs. Using WGCNA we predicted cis targets of lncRNAs, and the results suggested that lncRNAs may be involved in regulating genes that play a key role in ethylene-induced flowering. Further studies is needed to uncover the functions of the lncRNAs whose expression changed by ethylene treatment.

DNA methylation has been extensively studied in eukaryotes. DNA methylation is highly dynamic in response to environmental and endogenous cues. In rice, the DNA methylation level was increased by drought stress, and $70 \%$ of the changes were reset by removal of the drought stress ${ }^{35}$. Nitrogen deficiency can induce heritable alterations in DNA methylation to enhance tolerance among progeny in rice ${ }^{36}$. Ethylene is a plant hormone that regulates biotic and abiotic stress tolerance in plants ${ }^{6}$. Therefore, ethylene treatment may alter DNA methylation, as confirmed by our results. Compared with CK, the mean mCG and mCHG methylation level decreased in $\mathrm{HE}$ and but increased in LE, and $\mathrm{mCHH}$ methylation increased in both $\mathrm{HE}$ and LE. DMR analysis revealed that the upstream regions of 4,336 genes and the coding regions of 3,684 genes had overlapping DMRs in the mCG, $\mathrm{mCHG}$ and $\mathrm{mCHH}$ contexts in response to HE and LE. Moreover, in LE_vs_HE, the majority of DMRs were downregulated. For the genes in gene co-expression network, we identified 1,827, 1,705 and 412 genes interacted with HE, LE and CK separately overlapped with DMRs. In particular, for the candidate regulators in flowering related subnetworks, we identified 28 genes were overlapped with DMRs, including FTL1, FTL9, FTIP1, NAKR1, etc., of which the expression alternation may resulted by DNA methylation alteration during flowering inducing (Supplementary Dataset S3). Our results indicated that DNA methylation was differentially altered in response to $\mathrm{HE}$ and LE, which may play key role in ethylene-induced flowering.

Of the nine FT-like genes that were annotated in the pineapple genome, FTL1, FTL2, and FTL9 were induced by HE and LE. FTL4 was increased in LE and decreased in CK_vs_HE. Moreover, FTL6 was not expressed in our study but is known to be expressed in fresh and flower organs, indicating a role for FTL6 in floral organ development $^{14,16}$. Being increased in CK_vs_HE, FTL1, FTL2 and FTL9 may play overlapping or distinct roles in promoting flowering. Conversely, FTL4 was decreased in CK_vs_HE and may function as an antiflorigen. Our findings therefore support a balance between florigen and antiflorigen activity during flowering, as elucidated in tomato ${ }^{37}$.

After DEG analysis, we identified multiple genes related to flowering that may act as regulators of FTLs. The DEGs included homologs of genes that promote flowering, such as SPL14, BHLH93 ${ }^{38}$, MYB30 $0^{39-41}$, Flowering $B H L H(B H L H 78, B H L H 62 \text { and } B H L H 74)^{42}$, and $A S 1^{43}$, as well as homologs of genes that repress flowering, such as SPIN $1^{44}, R A V 1$ and $R A V^{45}, J M J 30$ and $E F M^{46}, N A C 35^{47}$ and $A P E T A L A 2^{48}$. In pineapple, SPL14-like is a homolog of OsSPL14 and AtSPL9, with OsSPL14 regulating tiller and panicle branching and AtSPL9 regulating flowering by increasing $\mathrm{miR} 172^{49}$. NaKR1 and FTL9 display similar expression patterns, regulating the long-distance translocation of $F T^{50}$. FTIP1, which contributes to the movement of FT from companion cells to sieve cells $s^{51}$, was also upregulated in CK_vs_HE. The upregulation of BHLH93, SPL14, AS1 and MYB30 and downregulation of JMJ30 and EFM in LE_vs_HE suggested these genes may play positive roles in pineapple flowering. Otherwise, TPS9 was upregulated in CK_vs_HE, along with the differential expression of SUS1, Carbonic anhydrase, phosphoenolpyruvate carboxylase, phosphoenolpyruvate carboxylase kinase, indicating the sugar pathway genes are also involved in flower induction.

In pineapple, flowering is triggered by a burst of ethylene production in response to various cues ${ }^{12}$. Ethylene treatment also triggers the biosynthesis of endogenous ethylene. The ethylene biosynthesis gene ACS7and ACO1 were upregulated after treatment, and the upregulation of ACO1 was also described by Espinosa et al. ${ }^{17}$. In the meanwhile, ethylene receptor genes ETR2 and ETR3, ethylene signaling genes EIL1 and EIL2 and ethylene responsive transcription factors ( $E R F 1, E R F 2, E R F 3$ etc.) were upregulated. The promoter regions of FTL1 and FTL9 contain ethylene response element (ERE)-boxes, and ethylene signaling may directly induce FTL1 and 
FTL9 expression. As the upregulation of homologs of auxin biosynthesis gene WEI2 and WEI ${ }^{52,53}$, gibberellin (GA) biosynthesis genes GA2ox8, abscisic acid (ABA) biosynthesis genes VP14 ${ }^{54,55}$, salicylic acid (SA) signaling regulator $N P R 1^{56}$ and the jasmonic acid (JA) signaling repressor $J A Z 1^{57}$ in both CK_vs_LE and CK_vs_HE, ethylene treatment may also alters JA, ABA, GA and auxin signaling or biosynthesis, which may be involved in flowering induction. The GA2ox8 were also upregulated in LE_vs_HE, indicating that the $\mathrm{GA}_{3}$ level reduced with the ethylene level increased during early stage of flowering inducing ${ }^{17}$. Besides, the GA2ox1 and ACS1 were not differently expressed as described by previous study, for the reason of difference of pineapple cultivars.

AS and APA increase the complexity of the transcriptome. HE and LE treatment induced specific isoforms and, meanwhile, the expression of some isoforms of genes was also altered. The transcripts of JAZ1 were terminated within the last exon, resulting in truncated transcripts lacking the Jas domain, which may have a contrary function in full-length transcripts in flowering timing ${ }^{58}$. Three alternative splice isoforms of FTIP1 were identified with two, three and four exons, and the isoforms with four exons was specifically induced by HE. Moreover, only the isoforms with specific APS of ACS1 was specifically induced by ethylene treatment.

In Arabidopsis, REPRESSOR OF SILENCING1 (ROS1) and DEMETER function as bifunctional DNA glycosylases/lyases and can activate DNA demethylation ${ }^{59,60}$. ROS4/IDM1 is involved in regulating active DNA demethylation ${ }^{61}$. The upregulation of homologs of ROS1, DEMETHER, and IDM1 by ethylene treatment suggests that ethylene may affect DNA methylation by regulating these enzymes. In addition to FTLs, many putative flowering-related genes, such as the homologs of CAL, NAC35, BHLH62, BHLH63, BHLH74, MIE1, PRR1, PRR95, APPR3, ARF23, SPL14, MYB30, PHYB, G2OX8, had overlapping DMRs, which may be involved in the regulation of these genes. In the meanwhile, the homolog of FTIP1 and NAKR1 that was responsible for the transport of $F T$. So, the DNA methylation alteration may participate in the regulation of flowering by regulated the activation and transport of FTLs.

lncRNAs may also directly regulate FTL expression. As shown in Fig. 6c, the antisense lncRNAs from FTL4 and FTL9 may also regulated the other FTLs in pineapple genome, for the highly similarity of the sequences of FTLs. Furthermore, the DNA methyltransferase DMT1, MYB30 that may directly activate FT expression, gibberellin biosynthesis gene GA2OX8 and ethylene signaling gene EIL1 were predicted to be targeted by lncRNAs in cis. For the limit information of pineapple genome, the functions of the most lncRNAs were unclear, some of which may directly or indirectly regulate FTLs.

In our study, LE treatment did not induce pineapple flowering, whereas HE treatment can induce flowering sufficiently. Similarly, in Guzmania lingulata, another bromeliad, plants treated with ethylene treatment for $4 \mathrm{~h}$ (no flowering) and $6 \mathrm{~h}$ (flowering) showed sharply different results ${ }^{62}$. Thus, flowering induction is thought to require a signaling threshold. LE treatment did not induce the threshold level of ethylene response factors. Continuous LE treatment can also induce flowering, and LE treatment is thought to induce the initial response, while HE induces the subsequent response. Through differential expression analysis and co-expression analysis, we detected the key flowering-related genes and their co-expression network, in which FTLs may play a central role, while the $L F Y$ and SOC1 were not differently expressed. The dose-dependent induction of FTLs may be a key message that contributes to dose-dependent flowering induction. Through differential methylation region analysis, we also detected thousands of DMRs that contribute to dose-dependent flowering induction. As flowering integrators, FTLs may be controlled by multiple flowering promoters and repressors, including protein-coding genes, lncRNAs and DNA methylation.

\section{Conclusion}

In the present study, we applied iso-seq and Illumina short-reads sequencing for transcriptome analysis. Analysis of AS and APA using iso-seq reads unveiled the complexity of the transcriptome and improved the existing gene annotation of pineapple. WGBS revealed alterations to the DNA methylation landscape of pineapple in response to HE and LE. Two of the nine annotated FTL genes were differentially expressed in pineapple and may play distinct roles in ethylene-induced flowering. Altered DNA methylation, lncRNAs and multiple flowering-pathway genes may be involved in the regulation of FTLs in the early stage of flowering inducing. Our data will facilitate further studies of the mechanism of ethylene-induced flowering.

\section{Methods}

Plant materials and treatment. The pineapple cultivar 'Tainon 16' were planted with an accession number 1-77 in an experimental area of the Institute of Tropical Crop Genetic Resources, Chinese Academy of Tropical Agricultural Sciences (CATAS). Plants were treated with $20 \mathrm{~mL}$ of $600 \mathrm{mg} / \mathrm{L}$ ethephon solution, $20 \mathrm{~mL}$ of $1200 \mathrm{mg} / \mathrm{L}$ ethephon solution, or water as a control (CK). Treatments were carried out in triplicate. The white tissue of 'D' leaves and stem apex were sampled $24 \mathrm{~h}$ after treatment and immediately frozen in liquid nitrogen. The frozen tissues were sent to BGI Life Tech Co., Ltd (Shenzhen, China) for sequencing.

Bisulfite-treated library construction and sequencing. Genomic DNA was extracted from each sample using the CATB method ${ }^{63}$. DNA was fragmented to a mean size of 250 bp by sonication using a Bioruptor (Diagenode, Belgium). Using an Illumina TruSeq DNA Sample Prep Kit, the DNA fragments were then processed by repairing $3^{\prime}$ ends, adenylating $3^{\prime}$ ends, and ligating adaptors according to the manufacturer's instructions. The ligated DNA was converted with bisulfite using the EZ DNA Methylation Gold kit (ZYMO). Then, the different-sized inserts were excised from $2 \%$ TAE agarose gels. The products were purified using the QlAquik Gel Extraction kit (Qiagen), followed by PCR amplification. Finally, sequencing was performed on an Illumina Hiseq $^{\mathrm{TM}} 4000$ with paired-end 150 -bp reads.

Bisulfite sequencing data analysis. Raw bisulfite sequencing data were filtered by removing adaptor sequences, low-quality reads and contamination. The clean data were aligned to the pineapple genome using 
$\mathrm{BSMAP}^{64}$. After removing duplicate reads, the mapped reads were merged to calculate the counts of each potentially methylated cytosine. Differentially methylated regions (DMRs) in CG, CHG, and CHH contexts were detected using methylPipe, with default parameters for plants ${ }^{65}$.

RNA sample preparation, library construction and sequencing. Total RNA was extracted using a Trizol kit (Promega, USA) according the manufacturer's instructions. Total RNA was then treated with RNase-free DNase I (Takara Bio, Japan) to remove DNA. After quality verification using a 2100 Bioanalyzer and RNase-free agarose gel electrophoresis, the libraries were processed according the manufacturer's instructions of the Truseq. 2 RNA sample prep kit (Illumina, Inc. San Diego, CA, USA). The mRNA was isolated using oligo-dT beads (Qiagen) and the Poly (A) Purist ${ }^{\mathrm{TM}}$ Kit (Ambion, now Life Technologies). After quality verification on the 2100 Bioanalyzer, the products were fragmented and reverse transcribed to cDNA fragments using a PrimeScript 1st Strand cDNA Synthesis Kit (Takara). The products were then processed by end repair and adaptor ligation and subjected to sequencing using Illumina Hiseq ${ }^{\mathrm{TM}} 4000$.

The first- and second-strand cDNA were synthesized using the SMARTer PCR cDNA Synthesis Kit (Clontech) and Phusion High-Fidelity DNA Polymerase (NEB). Then, the size of the cDNA was selected with BluePipple (Sage Science), followed by normalization using the Trimmer- 2 cDNA Normalization Kit (Evrogen) and amplification. Four fractions with normalized cDNA with sizes of $<1,1-2,2-3$, and $>3 \mathrm{~kb}$ were processed to SMRT cell libraries using the DNA Template Prep Kit (Pacific Biosciences of California, Inc.). Following binding of V2 primers and SA-DNA polymerase to the templates, the complexes were then bound to magnetic beads for sequencing on PacBio RS II and Sequel. Libraries with cDNA sizes $<1$ and $>3 \mathrm{~kb}$ were sequenced with two cells and the other libraries with one cell on Sequel. For low-yield libraries with $<1 \mathrm{~kb}$-size cDNA, the libraries were also sequenced on PacBio RS II with three cells.

Using the SMART Analysis Server 2.2.0 (Pacific Biosciences of California, Inc.), the raw data were filtered to generate reads of insert (ROIs), followed by classification of ROIs as full-length and non-full-length reads. Full-length reads were clustered using Iterative Clustering for Error (ICE) Correction and subsequently polished using Quiver. The polished full-length and high-quality sequences were mapped to the genome using GAMP ${ }^{66}$. Using Cuffcompare ${ }^{67}$, the reads were compared to the genome annotation, and then AS isoforms and APA isoforms were obtained. Then, the coding potentiality of the remaining reads was predicted using $\mathrm{CPC}^{68}$, $\mathrm{CPAT}^{69}$ and PLEK $^{54}$. According the results, novel coding and non-coding transcripts were predicted. In addition, reads that mapped to miRNA and phasi-siRNA loci were also extracted.

To predict lncRNA targets, we predicted interaction among lncRNAs and genes and the interaction with a score $>0.2$ were retained. LncRNA located within the $10 \mathrm{~kb}$ upstream and $100 \mathrm{~kb}$ downstream of gene and interacted with gene in the co-expression network were supposed to target at the gene.

Network analysis. The gene co-expression network was inferred using WGCNA ${ }^{55}$. Based on the gene expression data, gene clusters and relationships among clusters were detected use SPINE ${ }^{70}$. The network was visualized with Cytoscape v3.4. Genes were prioritized based on the DISCERN score to determine which genes contributed to topological changes of the gene-regulator-dependence network ${ }^{71}$.

qRT-PCR validation. We selected 18 protein-coding genes and 14 lncRNAs for qRT-PCR validation. The primers were designed with PRIMER 6.0 software (University of Plymouth) and listed in Supplementary Table S4 and Table S5. The Actin-2 (XM_020227265.1) was used as an endogenous control. For each sample, the reaction mixture for qRT-PCR consisted of $5 \mu \mathrm{L}$ of $2 \times$ SYBR Green Master Mix Reagent (Applied Biosystems), $0.2 \mu \mathrm{M}$ gene-specific primers and $50 \mathrm{ng}$ of the cDNA sample. The amplification reactions were performed as follows: $95^{\circ} \mathrm{C}$ for $10 \mathrm{~min}$ and $45 \mathrm{cycles}$ of $95^{\circ} \mathrm{C}$ for $5 \mathrm{~s}$ and $60^{\circ} \mathrm{C}$ for $30 \mathrm{~s}$. The relative expression of each gene was calculated using the $2-\Delta \Delta$ Ct method. qRT-PCR was performed with 3 biological replicates and 3 technical replicates for each experiment.

Availability of data and materials. Raw sequences of this study were deposited in NCBI SRA database SRP111498. The iso-seq produced high-quality sequences are available in accession SRR5816603. The Illumina short-reads are available in accession SRR5816604- SRR5816611 and SRR5816613 and WGBS produced reads are available in accession SRR5816601- SRR5816602 and SRR5816613.

\section{References}

1. Bartholomew, D. P., Paull, R. E. \& Rohrbach, K. G. The pineapple: botany, production and uses. (CABI Publishing, 2003).

2. Greenup, A., Peacock, W. J., Dennis, E. S. \& Trevaskis, B. The molecular biology of seasonal flowering-responses in Arabidopsis and the cereals. Annals of Botany 103, 1165-1172 (2009).

3. Amasino, R. Seasonal and developmental timing of flowering. Plant Journal 61, 1001-1013 (2010).

4. Wang, J. W. Regulation of flowering time by the miR156-mediated age pathway. Journal of Experimental Botany 65, 4723-4730 (2014).

5. Blümel, M., Dally, N. \& Jung, C. Flowering time regulation in crops - what did we learn from Arabidopsis? Current Opinion in Biotechnology 32C, 121-129 (2014).

6. Kazan, K. Diverse roles of jasmonates and ethylene in abiotic stress tolerance. Trends in plant science 20, 219-229 (2015).

7. Wen, C. K. et al. Ethylene in Plants. (Springer Netherlands, 2015).

8. Achard, P. et al. The plant stress hormone ethylene controls floral transition via DELLA-dependent regulation of floral meristemidentity genes. Proceedings of the National Academy of Sciences 104, 6484-6489 (2007).

9. Ogawara, T., Higashi, K., Kamada, H. \& Ezura, H. Ethylene advances the transition from vegetative growth to flowering in Arabidopsis thaliana. Journal of Plant Physiology 160, 1335-1340 (2003).

10. Wuriyanghan, H. et al. The ethylene receptor ETR2 delays floral transition and affects starch accumulation in rice. Plant Cell 21, 1473-1494 (2009).

11. Wang, Q. Rice CONSTITUTIVE TRIPLE-RESPONSE2 is involved in the ethylene-receptor signalling and regulation of various aspects of rice growth and development. Journal of Experimental Botany 64, 4863-4875 (2013). 
12. Trusov, Y. \& Botella, J. R. Silencing of the ACC synthase gene ACACS2 causes delayed flowering in pineapple [Ananas comosus (L.) Merr.]. Journal of experimental botany 57, 3953-3960 (2006).

13. Li, Y. H. et al. Molecular Cloning and Characterization of Four Genes Encoding Ethylene Receptors Associated with Pineapple (Ananas comosusL.) Flowering. Frontiers in Plant Science 7 (2016).

14. Lv, L. L. et al. Isolation and characterization of a FLOWERING LOCUS T homolog from pineapple (Ananas comosus (L.) Merr). Gene 505, 368-373 (2012).

15. Lv, L. L. et al. Cloning and Expression Analysis of a PISTILLATA Homologous Gene from Pineapple (Ananas comosus L. Merr). International Journal of Molecular Sciences 13, 1039-1053 (2011).

16. Liu, C. H. \& Fan, C. De novoTranscriptome Assembly of Floral Buds of Pineapple and Identification of Differentially Expressed Genes in Response to Ethephon Induction. Frontiers in Plant Science 7 (2016).

17. Ávila, M. et al. Early histological, hormonal, and molecular changes during pineapple (Ananas comosus (L.) Merrill) artificial flowering induction. Journal of Plant Physiology, 11-19 (2016).

18. Yang, H. et al. Whole-genome DNA methylation patterns and complex associations with gene structure and expression during flower development in Arabidopsis. Plant Journal 81, 268-281 (2014).

19. Dowen, R. H. \& Ecker, J. R. Widespread dynamic DNA methylation in response to biotic stress. Proceedings of the National Academy of Sciences of the United States of America 109, 2183-2191 (2012).

20. Trionnaire, G. L. L. \& Twell, D. Small RNAs in angiosperm gametophytes: from epigenetics to gamete development. Genes \& Development 24, 1081-1085 (2010).

21. Xing, M. Q. et al. Global Analysis Reveals the Crucial Roles of DNA Methylation during Rice Seed Development. Plant Physiology 168, 1417-1432 (2015).

22. Zhong, S. et al. Single-base resolution methylomes of tomato fruit development reveal epigenome modifications associated with ripening. Nature Biotechnology 31, 154-159 (2013).

23. Kondo, H., Miura, T., Wada, K. C. \& Takeno, K. Induction of flowering by 5 -azacytidine in some plant species: relationship between the stability of photoperiodically induced flowering and flower-inducing effect of DNA demethylation. Physiologia Plantarum 131, $462-469$ (2007).

24. Finnegan, E. J., Genger, R. K., Kovac, K., Peacock, W. J. \& Dennis, E. S. DNA methylation and the promotion of flowering by vernalization. Proceedings of the National Academy of Sciences of the United States of America 95, 5824-5829 (1998).

25. Brock, R. D. \& Davidson, J. L. 5-azacytidine and gamma rays partially substitute for cold treatment in vernalizing winter wheat. Environmental \& Experimental Botany 34, 195-199 (1994).

26. Redwan, R. M., Saidin, A. \& Kumar, S. V. The draft genome of MD-2 pineapple using hybrid error correction of long reads. Dna Research An International Journal for Rapid Publication of Reports on Genes \& Genomes (2016).

27. Ming, R. et al. The pineapple genome and the evolution of CAM photosynthesis. Nature Genetics 47, 1435-1442 (2015).

28. Jin, J. et al. PlantTFDB 4.0: toward a central hub for transcription factors and regulatory interactions in plants. Nucleic Acids Research 45, D1040-D1045 (2016).

29. Meng, X., Muszynski, M. G. \& Danilevskaya, O. N. The FT-like ZCN8 Gene Functions as a Floral Activator and Is Involved in Photoperiod Sensitivity in Maize. Plant Cell 23, 942-960 (2011).

30. Lee, R., Baldwin, S., Kenel, F., Mccallum, J. \& Macknight, R. FLOWERING LOCUS T genes control onion bulb formation and flowering. Nature Communications 4, 2884-2884 (2013).

31. Komiya, R., Ikegami, A., Tamaki, S., Yokoi, S. \& Shimamoto, K. Hd3a and RFT1 are essential for flowering in rice. Development 135, $767-774$ (2008)

32. Gallart, A. P., Pulido, A. H., Sanseverino, W. \& Cigliano, R. A. GREENC: a Wiki-based database of plant lncRNAs. Nucleic Acids Research 44, D1161-D1166 (2015).

33. Heo, J. B. \& Sung, S. Vernalization-mediated epigenetic silencing by a long intronic noncoding RNA. Science 331, 76-79 (2011).

34. Wang, B. et al. Unveiling the complexity of the maize transcriptome by single-molecule long-read sequencing. Nature Communications 7, 11708 (2016).

35. Wang, W. S. et al. Drought-induced site-specific DNA methylation and its association with drought tolerance in rice (L.).

36. Kou, H. P. et al. Heritable alteration in DNA methylation induced by nitrogen-deficiency stress accompanies enhanced tolerance by progenies to the stress in rice (Oryza sativa L.). Journal of Plant Physiology 168, 1685-1693 (2011).

37. Kai, C. et al. Four Tomato FLOWERING LOCUS T-Like Proteins Act Antagonistically to Regulate Floral Initiation. Frontiers in Plant Science 6, 14-17 (2016).

38. Sharma, N. et al. NO FLOWERING IN SHORT DAY (NFL) is a bHLH transcription factor that promotes flowering specifically under short-day conditions in Arabidopsis. Development 143, págs. 200501-201100 (2016).

39. Liu, L. et al. Elevated Levels of MYB30 in the phloem accelerate flowering in Arabidopsis through the regulation of FLOWERING LOCUS T. PloS one 9 , e89799 (2014).

40. Zheng, Y., Schumaker, K. S. \& Guo, Y. Sumoylation of transcription factor MYB30 by the small ubiquitin-like modifier E3 ligase SIZ1 mediates abscisic acid response in Arabidopsis thaliana. Proceedings of the National Academy of Sciences 109, 12822-12827 (2012).

41. Raffaele, S., Rivas, S. \& Roby, D. An essential role for salicylic acid in AtMYB30-mediated control of the hypersensitive cell death program in Arabidopsis. FEBS letters 580, 3498-3504 (2006).

42. Ito, S. et al. FLOWERING BHLH transcriptional activators control expression of the photoperiodic flowering regulator CONSTANS in Arabidopsis. Proceedings of the National Academy of Sciences of the United States of America 109, 3582 (2012).

43. Song, Y. H., Lee, I., Lee, S. Y., Imaizumi, T. \& Hong, J. C. CONSTANS and ASYMMETRIC LEAVES 1 complex is involved in the induction of FLOWERING LOCUS T in photoperiodic flowering in Arabidopsis. The Plant Journal 69, 332-342 (2012).

44. Vega-Sánchez, M. E., Zeng, L., Chen, S., Leung, H. \& Wang, G.-L. SPIN1, a K homology domain protein negatively regulated and ubiquitinated by the E3 ubiquitin ligase SPL11, is involved in flowering time control in rice. The Plant Cell 20, 1456-1469 (2008).

45. Mittal, A., Jiang, Y., Ritchie, G. L., Burke, J. J. \& Rock, C. D. AtRAV1 and AtRAV2 overexpression in cotton increases fiber length differentially under drought stress and delays flowering. Plant Science 241, 78-95 (2015).

46. Yan, Y. et al. A MYB-domain protein EFM mediates flowering responses to environmental cues in Arabidopsis. Developmental Cell 30, 437 (2014).

47. Yoo, S. Y., Kim, Y., Kim, S. Y., Lee, J. S. \& Ji, H. A. Control of Flowering Time and Cold Response by a NAC-Domain Protein in Arabidopsis. Plos One 2, e642 (2007).

48. Aukerman, M. J. \& Sakai, H. Regulation of flowering time and floral organ identity by a microRNA and its APETALA2-like target genes. The Plant Cell 15, 2730-2741 (2003)

49. Wang, L. et al. Coordinated regulation of vegetative and reproductive branching in rice. Proceedings of the National Academy of Sciences 112, 15504-15509 (2015).

50. Zhu, Y., Liu, L., Shen, L. \& Yu, H. NaKR1 regulates long-distance movement of FLOWERING LOCUS T in Arabidopsis 2 (2016).

51. Liu, L. et al. FTIP1 is an essential regulator required for florigen transport. Plos Biology 10, e1001313 (2012).

52. Stepanova, A. N. et al. TAA1 -Mediated Auxin Biosynthesis Is Essential for Hormone Crosstalk and Plant Development. Cell 133, 177-191 (2008).

53. Stepanova, A. N., Yun, J., Likhacheva, A. V. \& Alonso, J. M. Multilevel Interactions between Ethylene and Auxin in Arabidopsis Roots. Plant Cell 19, 2169-2185 (2007). 
54. Li, A., Zhang, J. \& Zhou, Z. PLEK: a tool for predicting long non-coding RNAs and messenger RNAs based on an improved k-mer scheme. BMC Bioinformatics 15, 1-10 (2014).

55. Langfelder, P. \& Horvath, S. WGCNA: an R package for weighted correlation network analysis. Bmc Bioinformatics 9, 1-13 (2008).

56. Wang, G.-F. et al. Multiple roles of WIN3 in regulating disease resistance, cell death, and flowering time in Arabidopsis. Plant physiology 156, 1508-1519 (2011).

57. Thines, B. et al. JAZ repressor proteins are targets of the SCF(COI1) complex during jasmonate signalling. Nature 448, 661 (2007).

58. Messing, S. A. J. \& Amzel, L. M. Structural Insights into Maize Viviparous14, a Key Enzyme in the Biosynthesis of the Phytohormone Abscisic Acid. Plant Cell 22, 2970-2980 (2010).

59. Gong, Z. et al. ROS1, a Repressor of Transcriptional Gene Silencing in Arabidopsis, Encodes a DNA Glycosylase/Lyase. Cell 111, 803-814 (2002).

60. Gehring, M. et al. DEMETER DNA Glycosylase Establishes MEDEA Polycomb Gene Self-Imprinting by Allele-Specific Demethylation. Cell 124, 495-506 (2011).

61. Qian, W. et al. Regulation of Active DNA Demethylation by an $\alpha$-Crystallin Domain Protein in Arabidopsis. Molecular Cell 55, 361-371 (2014).

62. Dukovski, D., Bernatzky, R. \& Han, S. Flowering induction of Guzmania by ethylene. Scientia Horticulturae 110, 104-108 (2006).

63. Paterson, A. H., Brubaker, C. L. \& Wendel, J. F. A rapid method for extraction of cotton (Gossypium Spp.) genomic DNA suitable for RFLP or PCR analysis. Plant Molecular Biology Reporter 11, 122-127 (1993).

64. Yuanxin, X. W. L. BSMAP: whole genome bisulfite sequence MAPping program. Bmc Bioinformatics 10, 232-232 (2009).

65. Kishore, K. et al. methylPipe and compEpiTools: a suite of R packages for the integrative analysis of epigenomics data. Bmc Bioinformatics 16, 1-11 (2015).

66. Wu, T. D. \& Watanabe, C. K. GMAP: a genomic mapping and alignment program for mRNA and EST sequences. Bioinformatics 21, 1859-1875 (2005).

67. C, T. et al. Transcript assembly and quantification by RNA-Seq reveals unannotated transcripts and isoform switching during cell differentiation. Nature Biotechnology 28, 511-515 (2010).

68. Kong, L. et al. CPC: assess the protein-coding potential of transcripts using sequence features and support vector machine. Nucleic Acids Research 69, 1-13 (2007).

69. Wang, L. et al. CPAT: Coding-Potential Assessment Tool using an alignment-free logistic regression model. Nucleic Acids Research 41, e74-e74 (2013).

70. Mansfeldt, C. B. SPINE: SParse eIgengene NEtwork Linking Gene Expression Clusters in Dehalococcoides mccartyi to Perturbations in Experimental Conditions. Plos One 10 (2015).

71. Grechkin, M., Logsdon, B. A., Gentles, A. J. \& Lee, S. I. Identifying Network Perturbation in Cancer. Plos Computational Biology 12 (2016).

\section{Acknowledgements}

This study was funded by the Natural Science Foundation of China (NSFC) (Item number: 31372106, 31601793).

\section{Author Contributions}

Z.Y.L. and L.X. and J.B.W. conceived and designed the experiments and wrote the papers; J.B.W. and J.J.Z. and Y.L.F. and M.L. and M.F.A. performed the experiments and analyzed the data; Y.L.F. and M.L. contributed reagents/materials/analysis tools. All authors read and approved the final manuscript.

\section{Additional Information}

Supplementary information accompanies this paper at https://doi.org/10.1038/s41598-017-17460-5.

Competing Interests: The authors declare that they have no competing interests.

Publisher's note: Springer Nature remains neutral with regard to jurisdictional claims in published maps and institutional affiliations.

(c) (i) Open Access This article is licensed under a Creative Commons Attribution 4.0 International

License, which permits use, sharing, adaptation, distribution and reproduction in any medium or format, as long as you give appropriate credit to the original author(s) and the source, provide a link to the Creative Commons license, and indicate if changes were made. The images or other third party material in this article are included in the article's Creative Commons license, unless indicated otherwise in a credit line to the material. If material is not included in the article's Creative Commons license and your intended use is not permitted by statutory regulation or exceeds the permitted use, you will need to obtain permission directly from the copyright holder. To view a copy of this license, visit http://creativecommons.org/licenses/by/4.0/.

(c) The Author(s) 2017 